\title{
Malaria surveillance from both ends: concurrent detection of Plasmodium falciparum in saliva and excreta harvested from Anopheles mosquitoes
}

\author{
Ana L. Ramírez ${ }^{1,2^{*}}$, Andrew F. van den Hurk ${ }^{3}$, lan M. Mackay ${ }^{3}$, Annie S. P. Yang ${ }^{4,5,6}$, Glen R. Hewitson ${ }^{3}$,
} Jamie L. McMahon ${ }^{3}$, Justin A. Boddey ${ }^{4,5}$, Scott A. Ritchie ${ }^{1,2}$ and Sara M. Erickson ${ }^{4,5}$

\begin{abstract}
Background: Malaria is the most important vector-borne disease in the world. Epidemiological and ecological studies of malaria traditionally utilize detection of Plasmodium sporozoites in whole mosquitoes or salivary glands by microscopy or serological or molecular assays. However, these methods are labor-intensive, and can over- or underestimate mosquito transmission potential. To overcome these limitations, alternative sample types have been evaluated for the study of malaria. It was recently shown that Plasmodium could be detected in saliva expectorated on honeysoaked cards by Anopheles stephensi, providing a better estimate of transmission risk. We evaluated whether excretion of Plasmodium falciparum nucleic acid by An. stephensi correlates with expectoration of parasites in saliva, thus providing an additional sample type for estimating transmission potential. Mosquitoes were exposed to infectious blood meals containing cultured gametocytes, and excreta collected at different time points post-exposure. Saliva was collected on honey-soaked filter paper cards, and salivary glands were dissected and examined microscopically for sporozoites. Excreta and saliva samples were tested by real time polymerase chain reaction (RT-rtPCR).
\end{abstract}

Results: Plasmodium falciparum RNA was detected in mosquito excreta as early as four days after ingesting a bloodmeal containing gametocytes. Once sporogony (the development of sporozoites) occurred, P. falciparum RNA was detected concurrently in both excreta and saliva samples. In the majority of cases, no difference was observed between the $C_{\mathrm{t}}$ values obtained from matched excreta and saliva samples, suggesting that both samples provide equally sensitive results. A positive association was observed between the molecular detection of the parasites in both samples and the proportion of mosquitoes with sporozoites in their salivary glands from each container. No distinguishable parasites were observed when excreta samples were stained and microscopically analyzed.

Conclusions: Mosquito saliva and excreta are easily collected and are promising for surveillance of malaria-causing parasites, especially in low transmission settings or in places where arboviruses co-circulate.

Keywords: Malaria, Mosquito, Saliva, Excreta, Sporozoite, Plasmodium falciparum, Anopheles stephensi

\section{Background}

Malaria is the deadliest vector-borne disease, with an estimated 219 million cases and 435,000 deaths in 2017

\footnotetext{
*Correspondence: ana.ramirez1@my.jcu.edu.au

${ }^{2}$ Australian Institute of Tropical Health and Medicine, James Cook University, PO Box 6811, Cairns, QLD 4870, Australia

Full list of author information is available at the end of the article
}

alone [1]. More than $90 \%$ of the cases occur in sub-Saharan Africa, and children under five years are the most vulnerable group. Plasmodium falciparum is the most prevalent causative agent of human malaria and has the most severe clinical manifestations [2]. The parasites are transmitted to humans by anopheline mosquitoes. More than 70 Anopheles species are competent vectors of malaria and more than half of these are responsible for 
transmitting the majority of human malaria parasites [3, 4].

Malaria control, elimination and ultimately, eradication, are global priorities, with 21 countries committed to eliminate malaria by 2020 [5]. Malaria elimination is achieved through a combination of antimalarial treatments (such as artemisinin-based combination therapy, $\mathrm{ACT}$ ), vector control and source reduction of mosquito larval habitats. Surveillance is a crucial component of malaria intervention programmes, providing information to guide initiatives and measure their impact [6] and is regarded as one of the three fundamental pillars of the Global Technical Strategy [7]. Malaria surveillance strategies are dependent on the level of transmission, where lower levels of transmission require increased efforts to detect new cases and transmission foci. Generally, malaria surveillance focuses on passive or active case detection, monitoring of anti-malarial drug resistance and entomological surveillance, including detection of insecticide resistance [8].

Detection of Plasmodium in mosquitoes is an essential parameter used to estimate metrics of exposure and transmission intensity. The sporozoite, the infectious stage of the parasite in the mosquito is usually the target of these efforts. There are several approaches to detect sporozoites in field-collected mosquitoes. Traditionally, their salivary glands are dissected and observed under a compound microscope for the presence of sporozoites $[9,10]$. Alternatively, enzyme-linked immunosorbent assays (ELISAs) have been used to detect sporozoite protein in salivary glands or pools of mosquitoes [11, 12]. Rapid diagnostic tests in dipstick format have also been developed [13, 14], with results comparable to those obtained by ELISA [15]. A variety of polymerase chain reaction (PCR) methods are available, with high sensitivity and versatility [16-18]. However, although certainly useful, these techniques have limitations. Dissection and observation of salivary glands is time-consuming, require skill and expertise, can fail to detect infections with low numbers of sporozoites and is not species specific. Immunoassays can yield false positives that need to be confirmed by molecular methods $[10,19,20]$. Even though PCR assays allow for highthroughput analysis, the identification and sorting of mosquitoes can be labor-intensive, especially for larger collections. They also require specialized facilities, equipment and expertise, which is often not available in low resource settings. Finally, all these methods can overestimate transmission, since not all the sporozoites present in the salivary glands will be ejected by a feeding mosquito [21].

Analysis of mosquito saliva for the presence of pathogens provides a better estimate of transmission risk.
Mosquito saliva has been used for the study of other mosquito-borne diseases, particularly arthropod-borne viruses (arboviruses), both in the field and the laboratory [22, 23]. It had been demonstrated that P. falciparum could be detected in mosquito saliva collected by forced salivation [9], but it was not until recently that $P$. falciparum sporozoites were detected in saliva expectorated on honey-soaked nucleic acid preservation cards, allowing for detection without killing the mosquito [24]. Alternatively, mosquito excreta has emerged as a promising sample for the study of arboviruses [25-27], filarial parasites and malaria [28]. Mosquito excreta has the added potential to be used for xenomonitoring, where the mosquitoes are used as "flying syringes" to sample vertebrate hosts to monitor human and animal diseases and methodologies are being developed to collect mosquito excreta in the field [29].

The primary objective of the current study was to determine, through proof of concept, if $P$. falciparum could be detected by molecular assays concurrently in excreta and saliva of Anopheles stephensi mosquitoes. We also correlated the detection of the parasite in excreta and saliva with salivary gland sporozoite infection in the mosquitoes. Finally, we analyzed excreta samples microscopically for evidence of recognizable parasites.

\section{Methods}

\section{Parasite maintenance}

The asexual stages of $P$. falciparum NF54 were maintained at $4 \%$ hematocrit in human O-positive erythrocytes (Australian Red Cross, Melbourne) in RPMI-HEPES with $10 \%$ heat-inactivated human serum (Australian Red Cross, Melbourne) in an atmosphere of $94 \% \mathrm{~N}, 5 \% \mathrm{CO}_{2}$, $1 \% \mathrm{O}_{2}$ [30]. Gametocytes were generated as described previously, using the crash method [31]. After 17 days, gametocytes were quantified by Giemsa smears, harvested, and five different blood meals prepared by dilution to $0.3 \%$ stage $\mathrm{V}$ gametocytemia in human serum for feeding to mosquitoes [30].

\section{Mosquito rearing}

Experiments were performed using Anopheles stephensi mosquitoes (John Hopkins School of Public Health strain) at the Walter and Eliza Hall Institute of Medical Research. Larvae were fed a 1:1 ratio of TetraMin ${ }^{\circledR}$ and Nutrafin ${ }^{\circledR}$ Max tropical fish food flakes. After adult emergence, mosquitoes were provided sugar cubes and water in a cotton wick ad libitum. Females were offered mouse blood in water-jacketed, glass membrane feeders (Lillie Glassblowers, Inc., Georgia, USA) to stimulate egg production. All mosquitoes were maintained at $26^{\circ} \mathrm{C}, 80 \% \mathrm{RH}$ and 12:12 L:D for the duration of the study. 


\section{Exposure of mosquitoes to $P$. falciparum gametocyte cultures and analysis of parasite development}

Four- to five-day-old mosquitoes were deprived of sugar overnight $(10-14 \mathrm{~h})$ prior to being exposed to P. falciparum gametocytes. Females were aspirated into 0.946 1 paper cartons (Castaway Food Packaging, Australia) secured with mesh lids where they were offered a gametocytemic blood meal through a water-jacketed, glass membrane feeder. Two hours after feeding, mosquitoes were $\mathrm{CO}_{2}$ anesthetized and sorted on wet ice. Only fully engorged females were maintained, whilst males, non-fed and partially-fed females were discarded. Fully engorged females were immediately placed in a $24.5 \mathrm{~cm}^{3}$ mesh cage (Bugdorm-42222, Bugdorm, Taichung, Taiwan), with sugar cubes and a water wick, or in modified containers for excreta collection (see below). At day 8 post-exposure (PE), the midguts from 16-23 cold-anesthetized and ethanol-killed mosquitoes from each cohort were dissected and stained with $0.1 \%$ mercurochrome $(\mathrm{w} / \mathrm{v})$ in water, and oocysts per mosquito enumerated by microscopy. At day $17 \mathrm{PE}, 30-32$ salivary glands from mosquitoes from each cohort were dissected and pooled before being homogenized in PBS with a pestle to release sporozoites. After filtering through glass wool, sporozoites were counted using a Neubauer hemocytometer, and each cohort sample was counted in triplicate.

\section{Collection of mosquito excreta and saliva}

Two experiments were conducted to evaluate the use of mosquito excreta and saliva for P. falciparum detection. In the first experiment, groups of mosquitoes were followed over time to establish the time of first detection in excreta. For this, 20 batches of 5 mosquitoes which had been exposed to two different gametocytemic blood meals were placed in modified $150 \mathrm{ml}$ polypropylene containers for excreta collection [26]. The containers had a fiberglass insect screen floor to allow excreta to pass through onto a parafilm disc and the top opening of the containers was covered in mesh. Mosquitoes were maintained on cotton pledgets soaked in $15 \%$ honey water dyed with blue food coloring for excreta visualization. Excreta was collected daily from day 4 to $14 \mathrm{PE}$ using a cotton swab moistened with PBS. Swabs were placed in a $1.5 \mathrm{ml}$ tube with $500 \mu \mathrm{l}$ PBS and stored at $-80^{\circ} \mathrm{C}$. Cotton pledgets and parafilm discs were replaced daily to avoid cross-contamination and mortality was recorded daily.

In the second experiment, from day 15 to $19 \mathrm{PE}$, excreta and saliva were collected from groups of mosquitoes and the presence of sporozoites in their salivary glands was visually assessed. For this, 3 groups of 5 mosquitoes from cohorts that had fed on 5 different blood meals containing gametocytes were placed in modified containers as described above. For daily saliva collection, mosquitoes were allowed to feed on a $4 \mathrm{~cm}^{2}$ filter paper card (FP; low chamber filter paper, Bio-Rad Laboratories, California) soaked in $100 \%$ honey dyed with blue food coloring. After $24 \mathrm{~h}$, excreta was collected as previously described, whilst the FP cards were removed and placed in a $1.5 \mathrm{ml}$ tube containing $0.5 \mathrm{~mL}$ PBS and stored at $-80{ }^{\circ} \mathrm{C}$. The mosquitoes were $\mathrm{CO}_{2}$ anesthetized, ethanol-killed, and the salivary glands dissected and assessed for the presence of sporozoites using a compound microscope. The sporozoite rate of the container was calculated as the number of mosquitoes with sporozoites in their salivary glands per the number of surviving mosquitoes in the container.

\section{Detection of Plasmodium spp. by real-time RT-rtPCR}

Thawed excreta samples were agitated using a Qiagen Tissue Lyser II (Qiagen, Hilden, Germany) for 3 min at 26 $\mathrm{Hz}$ and centrifuged for $1 \mathrm{~min}$ at $14,000 \times g$ [26]. Thawed FP cards were maintained at $4{ }^{\circ} \mathrm{C}$ and briefly vortexed every $5 \mathrm{~min}$ for $20 \mathrm{~min}$ [22]. RNA was extracted from excreta samples and FP card eluates using a QIAmp OneFor-All Nucleic Acid Kit (Qiagen, Hilden, Germany) in a QIAxtractor (Qiagen, Hilden, Germany) according to the manufacturer's instructions. A Taqman ${ }^{\circledR}$ real-time RTPCR (RT-rtPCR) assay (modified from [32]) was used to detect Plasmodium spp. The assay amplifies a conserved region of the $18 S$ rRNA gene. The primers and probe were: forward primer (5'-AGG AAG TTT AAG GCA ACA ACA GGT- $3^{\prime}$ ); reverse primer (5'-GCA ATA ATC TAT CCC CAT CAC GA-3'); and probe (5'-6FAM-TGT CCT TAG ATG AAC TAG GCT GCA CGC G-BHQ-1$\left.3^{\prime}\right)$. Primer and probe oligonucleotides were synthesized by Sigma-Aldrich (Castle Hill, Australia). The reaction mix was prepared using SuperScript III Platinum ${ }^{\circledR}$ onestep quantitative RT-PCR system (Invitrogen, Carlsbad, CA) and contained $0.4 \mu \mathrm{l}$ of SuperScript ${ }^{\circledR}$ III/Platinum ${ }^{\circledR}$ Taq mix, $10 \mu \mathrm{l}$ of $2 \times$ reaction mix, $50 \mathrm{nM}$ of ROX reference dye, primers and probe in a final optimized concentration of $900 \mathrm{nM}$ and $150 \mathrm{nM}$ respectively, $5 \mu \mathrm{l}$ of extracted RNA and nuclease-free water to produce a final volume of $20 \mu \mathrm{l}$. The assays were run in a RotorGene 6000 real-time PCR cycler (Qiagen, Australia) with cycling conditions as follows: (i) one cycle at $50{ }^{\circ} \mathrm{C}$ for 5 min; (ii) one cycle at $95^{\circ} \mathrm{C}$ for $2 \mathrm{~min}$; and (iii) 50 cycles of $95{ }^{\circ} \mathrm{C}$ for $3 \mathrm{~s}$ and $60{ }^{\circ} \mathrm{C}$ for $30 \mathrm{~s}$. Each run included a positive extraction control (bovine viral diarrheal virus, BVDV) and a positive $P$. falciparum control extracted from sporozoites; a negative extraction control and a no-template control (molecular grade water). The cycle threshold number $\left(C_{t}\right)$ was determined for each sample; any sample with a $C_{t}>40$ was considered negative. 
To determine the assay's limit of detection, a sample of quantified sporozoites from salivary glands was extracted as described above, and 10-fold dilutions were used to generate a standard curve with undiluted RNA and each dilution $\left(10^{-1}\right.$ to $\left.10^{-8}\right)$ tested in triplicate.

\section{Visualization of $P$. falciparum in mosquito excreta}

A total of six aliquots from 10 excreta samples that were positive by RT-rtPCR were air-dried, methanol-fixed and dyed with $11 \%$ Giemsa stain diluted in distilled water for $60 \mathrm{~min}$ before being washed with water, dried and examined using a compound microscope under $1000 \times$ magnification.

\section{Statistical analyses}

All data sets were tested for normality using ShapiroWilks tests. Differences in salivary gland infection between cohorts were analyzed using a one-way ANOVA followed by Tukey's multiple post-hoc comparison test. Differences in oocyst counts between cohorts and $C_{t}$ values for excreta and expectorate between days and between groups were analyzed using the Kruskal-Wallis test followed by Dunn's multiple comparison test. Differences in $C_{t}$ values between excreta and saliva were analyzed using the Wilcoxon matched-pairs signed rank test. Differences between the proportion of positive saliva and excreta samples were analyzed using the Fisher's exact test. Associations between sporozoite rates and $C_{t}$ values from saliva and excreta were analyzed using Spearman's rank correlation. All figures, Kaplan-Meier survival curves and statistical analyses were performed using GraphPad Prism version 7.0c (GraphPad Software, La Jolla, CA, http://www.graphpad.com).

\section{Results}

\section{Parasite development in the mosquito}

There was no difference in mosquito survival distributions between cohorts (Log-Rank statistic $\chi_{(3)}^{2}=4.415$, $P=0.220$; Additional file 1: Figure S1). The overall oocyst rate (prevalence of mosquito infection) in mosquito midguts at day $8 \mathrm{PE}$ was $72.7 \%$, ranging from $55 \%$ for cohort C to $91 \%$ for cohort B (Fig. 1a). There was a significant difference between the median number of oocysts between groups (Kruskal-Wallis one-way ANOVA, $H_{(4)}$ $=15.67, P=0.0035$, Fig. 1b). On day $17 \mathrm{PE}$, the mean number of sporozoites per mosquito ranged from 2490 in cohort C to 9730 in cohort A (Fig. 1c). There was a significant difference between groups as determined by one-way ANOVA $\left(F_{(4,15)}=54.11, P<0.0001\right)$. However, post-hoc analysis showed that there was no significant difference in sporozoite load between some groups. Consequently, for further analyses of the second experiment, the cohorts were grouped as high sporozoite load (AE), mid sporozoite load (B) and low sporozoite load (CD) where applicable.

\section{Plasmodium falciparum sporozoite RT-rtPCR detection threshold}

To determine the RT-rtPCR assay's limit of detection, a standard curve was prepared using RNA extracted from quantified sporozoites purified from mosquito salivary glands on day 17 PE. Serial dilutions of parasite RNA resulted in an $R^{2}$ of 0.9451 and a slope of -2.92 , demonstrating the linear relationship between the logarithm of the number of parasites and $C_{t}$ value within a 4- $\log _{10}$ dynamic range (Additional file 2: Figure S2). At a $C_{t}$ value $>40$ P. falciparum could not be detected.
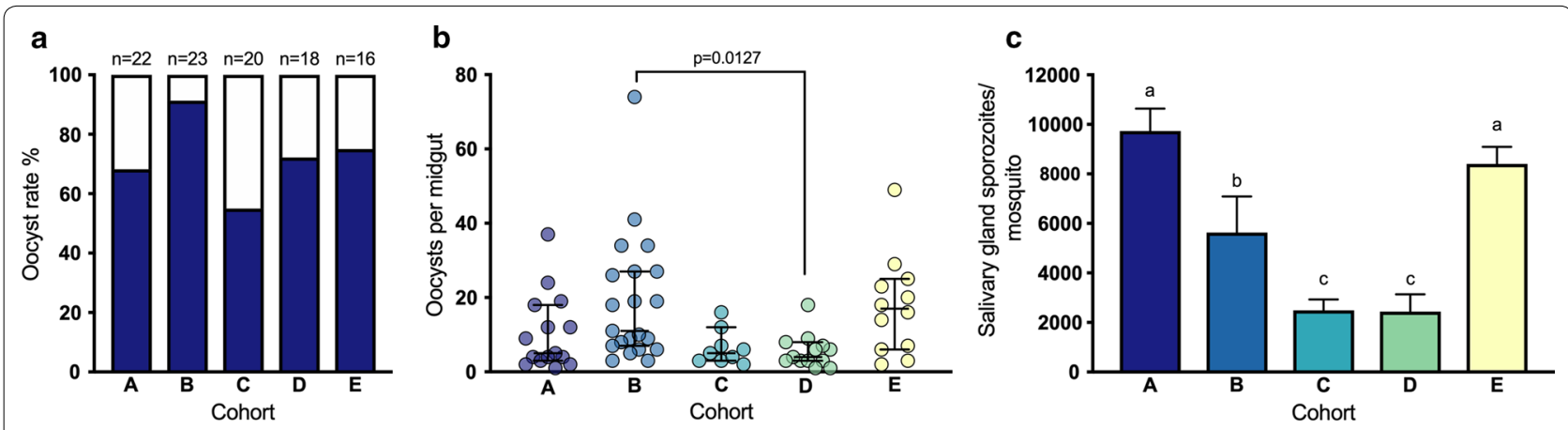

Fig. 1 Parasite development in mosquito cohorts exposed to five different bloodmeals (indicated as cohorts a-e). a Proportion of mosquitoes with oocysts in their midguts at day 8 PE. b Oocyst counts per mosquito midgut 8 days PE (median and 95\% Cl, Kruskal-Wallis one-way ANOVA followed by Dunn's multiple comparison test). Each dot corresponds to one midgut. c Salivary gland sporozoite loads per mosquito 17 days PE. Different letters indicate statistically significant differences between groups (mean $\pm \mathrm{SEM}, n=4$, one-way ANOVA test followed by Tukey's multiple comparison test, $P<0.05$ ) 


\section{Mosquitoes excrete $P$. falciparum material soon after ingesting an infectious blood meal}

The excreta from 10 containers from each of 20 original containers from the two cohorts was analyzed over time. Plasmodium falciparum was detected in mosquito excreta by RT-rtPCR as early as day 4 PE in both cohorts (Fig. 2). For cohort A (68.2\% oocyst rate and $9730 \pm 910$ sporozoites per mosquito), excreta samples collected from 8 out of 10 containers were positive at least once from day 4 to day $14 \mathrm{PE}$, with $10 \%(11 / 110)$ samples positive for $P$. falciparum overall. $C_{\mathrm{t}}$ values ranged from 27.5 to 37.9 . For cohort B $(91.3 \%$ oocyst rate and $5630 \pm 1460$ sporozoites per mosquito), excreta samples collected from 8 out of 10 containers were positive at least once for the duration of the experiment, with $16 \%$ (18/110) samples positive for the parasite and $\mathrm{C}_{\mathrm{t}}$ values ranging from 25.5 to 39.7. No statistically significant difference was observed between mean $C_{t}$ values between the cohorts (Two sample t-test, $t_{(2)}=0.5236, P=0.6048$ ). For both

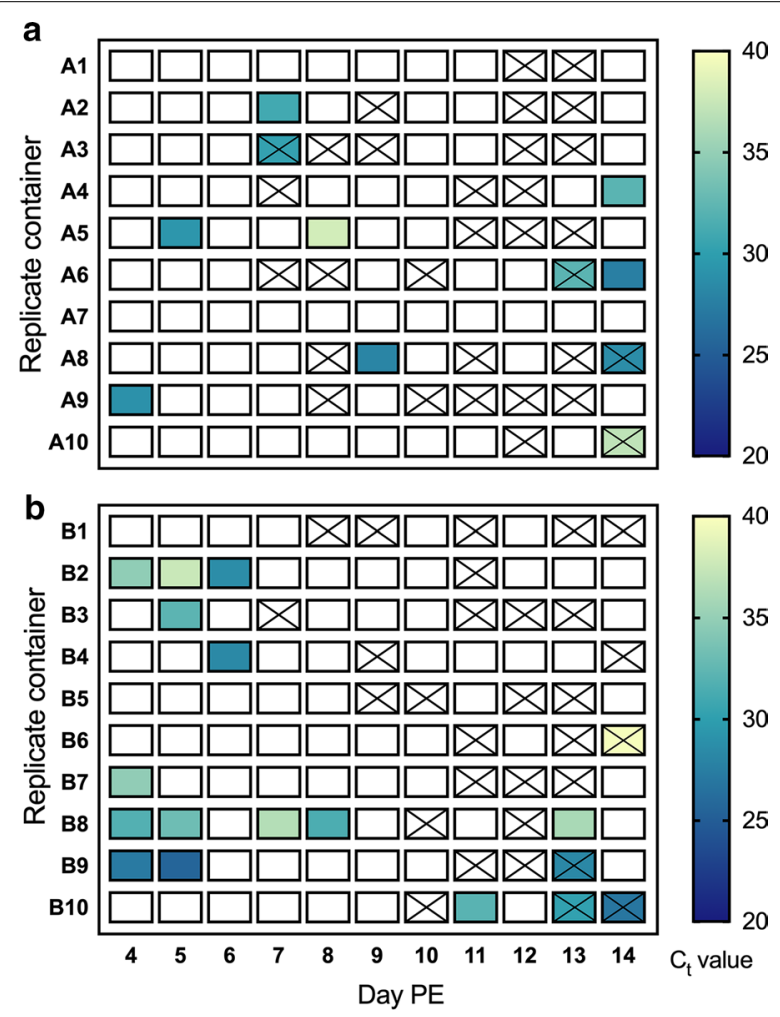

Fig. 2 Time series RT-rtPCR detection of $P$. falciparum in excreta from groups of 5 An. stephensi mosquitoes. Excreta was collected daily from day 4 to 14 post-exposure (PE). a Mosquitoes with 68.2\% oocyst rate and $9730 \pm 910$ sporozoites per mosquito. b Mosquitoes with

$91.3 \%$ oocyst rate and $5630 \pm 1460$ sporozoites per mosquito. Lower $C_{t}$ values correspond to a greater concentration of starting template; a blank cell indicates that P. falciparum RNA was not detected. An X indicates containers with no visible excreta spots cohorts, no positive samples were observed on day 10 and day $12 \mathrm{PE}$.

\section{Plasmodium falciparum can be detected concurrently in mosquito excreta and saliva after sporogony}

Plasmodium falciparum sporozoites were microscopically observed in the salivary glands of at least one mosquito removed from each of the containers analyzed in the second experiment (75/75). P. falciparum was detected by RT-rtPCR in $89 \%(67 / 75)$ of saliva samples and $91 \%(68 / 75)$ of excreta samples collected from day 15 to $19 \mathrm{PE}$, with no significant difference between these proportions (Fisher's exact test, $P>0.9999$ ). No significant differences were observed in median $C_{t}$ values of saliva samples between days within the same cohort (KruskalWallis one-way ANOVA, $P>0.05)$ or from excreta samples between days within the same cohort (KruskalWallis one-way ANOVA, $P>0.05)$. Thus, the samples from different days from the same cohort were analyzed together from this point onward. No statistically significant differences were observed between median $C_{t}$ values obtained from saliva and excreta, except the mosquitoes with a medium sporozoite load, where the median $C_{t}$ value was lower in excreta than saliva (Fig. 3a; 27.9 vs 30.0, Wilcoxon matched-pairs signed rank test $W_{(14)}=14$, $P=0.0134)$. When comparing detection between mosquitoes with different sporozoite loads, a statistically significant difference was observed in median $C_{t}$ values from saliva (Fig. 3b; Kruskal-Wallis one-way ANOVA, $\left.H_{(2)}=15.61, P=0.0004\right)$. A statistically significant difference was also observed in median $C_{t}$ values from excreta between these cohorts (Fig. 3c; Kruskal-Wallis one-way ANOVA, $\left.H_{(2)}=11.39, P=0.0034\right)$.

All containers from which saliva and excreta were harvested from had at least one mosquito with sporozoites in their salivary glands. The overall sporozoite rate for these mosquitoes was $60 \%$, and the sporozoite rates were $66 \%, 56 \%$ and $54 \%$ for high, mid and low sporozoite load cohorts, respectively. A negative association was observed between the sporozoite rate of the container and the $C_{t}$ value in saliva (Fig. 4a; Spearman's rank correlation $\left.\rho_{(65)}=-0.5408, P<0.0001\right)$ : the higher the proportion of mosquitoes with sporozoites in their salivary glands, the lower the $C_{t}$ value (indicating higher amounts of the template). For excreta, this association was lower but still negative (Fig. 4b; Spearman's rank correlation $\left.\rho_{(66)}=-0.3595, P=0.0026\right)$.

\section{Visualization of $P$. falciparum life stages in excreta samples} A subsample (10/68) of the excreta samples that were positive for $P$. falciparum by RT-rtPCR were examined microscopically in sextuplicate. No visual evidence of 


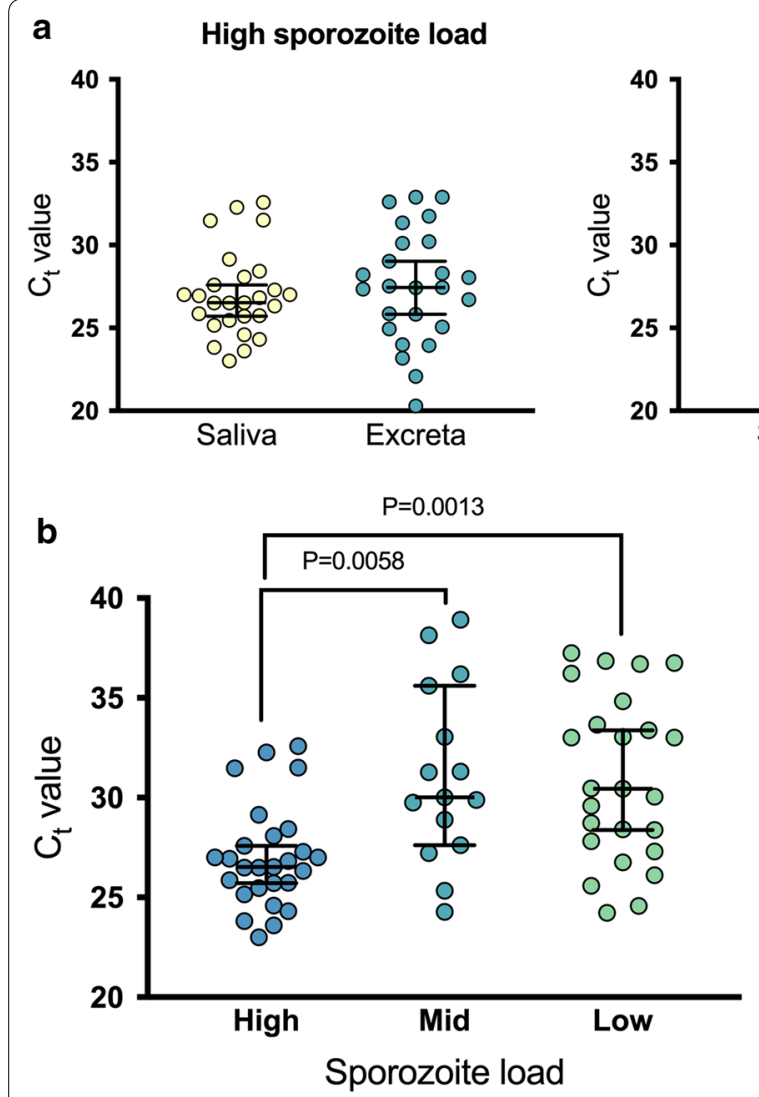

\section{Mid sporozoite load}

$\mathrm{P}=0.0134$



Low sporozoite load

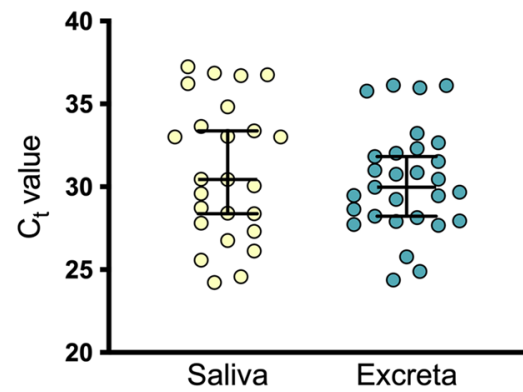

b

Fig. 3 RT-rtPCR detection of P. falciparum in mosquito secretions collected from mosquitoes with high, middle and low sporozoite loads on day 15 to 19 post-exposure. a Detection of $P$. falciparum in saliva vs excreta in mosquitoes from the cohorts with different sporozoite loads. Wilcoxon matched-pairs sign ranked test. Detection of P. falciparum in saliva (b) excreta (c) from mosquitoes with different sporozoite loads. Kruskal-Wallis one-way ANOVA with Dunn's multiple comparison test. Data are the median $C_{t}$ value $\pm 95 \% \mathrm{Cl}$. Each dot represents a group of 5 mosquitoes in a container. Lower $C_{t}$ values correspond to a greater concentration of starting template

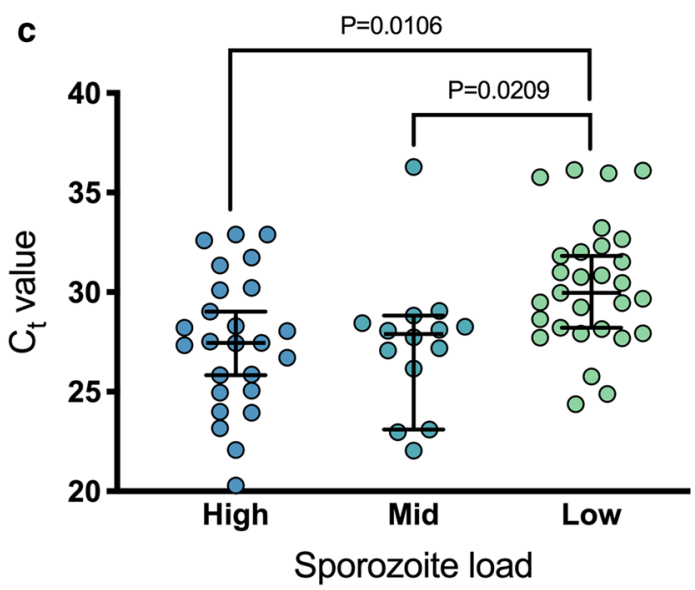


sporozoites or other life stage of the parasite was found in these samples.

\section{Discussion}

Given the limitations of traditional methods to study mosquito-borne diseases, there has been concern in finding innovative or alternative samples for analysis. Mosquito saliva expectorated during sugar feeding has been used for research and surveillance of arboviruses [22, 23 ], and recently mosquito excreta has been proposed as a sample to enhance the sensitivity of saliva detection or for molecular xenomonitoring [25-28]. To our knowledge, this is the first study to evaluate the excretion and expectoration of $P$. falciparum in parallel with parasite development in the mosquito. Our results confirm previous findings that Plasmodium can be detected in mosquito excreta [28] and saliva deposited on filter paper cards after sugar feeding [24].

Previous studies have demonstrated that $P$. falciparum DNA can be detected in mosquito excreta on days 2-3 PE [29]. In this study, excreta was not collected until day 4 PE, to allow for blood meal digestion, which takes approximately 72 hours to be completed [33]. Our results indicate that $P$. falciparum nucleic acid in mosquito excreta continues to be detectable after blood meal digestion from day 4 to at least $19 \mathrm{PE}$. The source of the nucleic acid or the parasite life stage in excreta is unknown, but several hypotheses could explain its presence. Once a mosquito feeds on an infected host, it ingests gametocytes, the sexual stage of the parasite. An hour later, fertilization occurs, and by 24 hours the ookinete enters the midgut were oocysts establish and begin mitosis [34]. It has been suggested that some of the material excreted in the early days could be metabolized merozoites [28], the asexual parasites in the intraerythrocytic cycle, which are present in ingested blood meal in a ratio of about 156 merozoites per gametocyte [35] and which cannot infect, or survive in, the mosquito.

Additionally, early stage parasite development from ookinete to oocyst is closely related with blood-meal digestion; ookinetes that fail to traverse the midgut and transform to oocysts after digestion are destroyed [36]. From day 11 to 16, the oocysts burst producing thousands of sporozoites that migrate through the hemocoel to the salivary glands [37]. This is an inefficient process: some of these oocysts may be unsuccessful in producing sporozoites and the released sporozoites can fail to navigate, invade or survive in the salivary glands, with less than $20 \%$ of the sporozoites released by oocysts reaching salivary glands [38]. The remaining sporozoites are degraded in the hemocoel [39], and although the mechanism is unknown, it is possible that the residue finds its way to the Malpighian tubules to be excreted with other unwanted substances of the hemolymph. Although $40 \%$ and $91 \%$ of the containers sampled from day 11 to 14 and 15 to 19 , respectively, were positive for $P$. falciparum by RT-rtPCR, no distinguishable parasites were observed under microscopy following Giemsa staining in any of the analyzed samples. Further studies of the contents of mosquito excreta are required to determine the source of the excreted nucleic acid.

We were able to detect $P$. falciparum sporozoites deposited on filter paper cards after sugar feeding on days 15 to $19 \mathrm{PE}$. Our results expand the results from Brugman et al., who detected sporozoites on cotton wool pledgets from day 18 to 24 [24]. It is likely that the differences in $C_{t}$ values in saliva samples between cohorts are due to differences in sporozoite rates and not in sporozoite loads. The high sporozoite load cohort also had the highest sporozoite rate (66\%) compared to the other cohorts (56\% and $54 \%$ for mid and low sporozoite cohorts respectively). This was further demonstrated by the negative association between the $C_{t}$ values obtained from saliva samples and the sporozoite rate of the container. Studies have suggested that the sporozoite load in the salivary glands is not a predictor for sporozoite transmission [40] because the structure of the salivary glands limits the number of sporozoites that are expectorated [21].

With the exception of the mid sporozoite load cohort, no significant differences were observed between detection of $P$. falciparum in excreta and saliva in samples collected after sporogony. It is interesting to note that although mosquitoes from this cohort had the highest oocyst rate $(91 \%)$ at day $8 \mathrm{PE}$, the sporozoite rate from day 15 to 19 was moderate $(56 \%)$ in the context of this experiment. This could explain the lower $C_{t}$ values observed in excreta, since many of the sporozoites produced by the oocysts may have failed to reach the salivary glands, and may have been destroyed and possibly voided in excreta.

In this study we did not directly evaluate the detection of the parasite in secretions from individual mosquitoes. However, we were able to detect $P$. falciparum in the saliva and excreta from $80 \%$ of containers where just one mosquito had a salivary gland infection, indicating that the method is sensitive enough to detect the parasites from an individual mosquito. Previous studies have demonstrated that trace amounts of Brugia malayi DNA are detectable in samples that contain excreta from as many as 500 uninfected mosquitoes [28]. Similarily, it does not appear that the saliva of numerous uninfected mosquitoes affects the detection of arboviruses in mosquito expectorate. It is unlikely that saliva or excreta from many mosquitoes would interfere with the detection of 
P. falciparum from a single infected mosquito. However this needs to be further evaluated.

Detection of Plasmodium in mosquito excreta and saliva has applications in the laboratory. Observation of oocysts in mosquito midguts can be used as an estimation of mosquito infectivity [41]; however, oocysts are not visible until 6-7 days after ingesting an infectious bloodmeal, making it impossible to determine the infectious status of mosquitoes for a week [36]. Our results suggest that excreta could be monitored after bloodmeal digestion as soon as day 4, allowing for an earlier estimation of the potential of the parasite to establish a midgut infection in a non-destructive manner. An important component of vectorial capacity is the estimation of the period of sporogony, the period from which a mosquito ingests gametocytes to when it can transmit sporozoites to a receptive host [42]. Traditionally, this has relied on the detection of sporozoites in mosquito salivary glands. Monitoring the expectoration of the parasite could be a useful tool for exploring genetic traits and different environmental conditions that influence this period, allowing for a precise measurement of time-to-event in individual mosquitoes [43]. Genetic analyses and drug and vaccine development studies often rely in infected mosquitoes feeding on animal models, such as mice $[44,45]$ and nonhuman primates [46, 47]. Since our method allows for non-destructive screening of the parasite in the vector, mosquitoes that are transmitting could be pre-selected to increases the chances of transmission and potentially reducing the number of animals used in an experiment.

The analysis of mosquito saliva and excreta could also be implemented for malaria surveillance in the field. Currently, parasite detection in mosquitoes requires testing thousands of mosquitoes, either individually by microscopy or in pools by ELISA or molecular methods. Indeed, as transmission of a pathogen decreases, larger numbers of mosquitoes are necessary to improve the likelihood of capturing the less frequent occurrence of infection. Honey-based surveillance using nucleic acid preservation cards or wicks to collect mosquito saliva has been successfully incorporated by public health agencies in Australia and USA for routine surveillance of arboviruses [23, $48,49]$ with several advantages over traditional methods. First, it reduces the number of samples that need to be processed down to 1-2 samples per trap. Secondly, the cards or wicks do not require a cold chain, making the method a logistically attractive approach. Finally, detection of the pathogen in mosquito saliva gives a better estimate of transmission risk, since only the mosquitoes that are transmitting will yield a positive result. Recently, it has been demonstrated that detection of arboviruses in excreta can be used to enhance the sensitivity of honeybased surveillance since the volume of the sample is larger $[25,26]$. In the context of malaria surveillance, honey-based methods could be incorporated in regions with known co-circulation of malaria and arboviruses with the advantage of detecting all the circulating pathogens from one sample. Since it is not possible to determine how many mosquitoes expectorated or excreted in a trap, it is not possible to calculate an entomological metric, such as the sporozoite rate. Additionally, although a positive excreta result would not be sufficient to suggest that the mosquitoes in the trap are transmitting Plasmodium, it would indicate that the parasite is circulating. However, together with geolocation and mapping of larval habitats and areas of human activity [50], a positive result can be used to identify potential foci of transmission. This is particularly interesting in low transmission settings or to monitor re-establishment after elimination. In this study we used RT-rtPCR for pathogen detection, but the use of portable and automated rapid diagnostic test (RDT) devices for detection of the parasite in mosquito saliva and excreta samples needs to be assessed. Although the majority of RDTs available for Plasmodium focus on diagnosis of human samples [51], a VecTest ${ }^{\mathrm{TM}}$ dipstick assay for detection of sporozoites from mosquitoes has been developed [13, 14]. Dipstick assays have the advantage of providing results within minutes and do not require specialized equipment or infrastructure. Currently, a centrifugal microfluidic multiplex vector-diagnostic platform (LabDisk) to be used with mosquitoes is being evaluated [52]. The sensitivity of these assays is not as good as PCR-based detection, but given that collection of mosquito saliva and excreta is relatively simple, it could be coupled with RDTs or portable devices for use in low-resource settings and remote locations.

\section{Conclusions}

The development of methods to estimate malaria transmission in low-transmission settings has been identified as one of the objectives by the malaria Eradication Research Agenda (malERA) [53]. As elimination targets are met, it is evident that novel approaches will be needed to ensure that transmission foci are identified, and re-establishment is prevented. Mosquito saliva and excreta have potential to be added to the array of samples supporting the crusade for malaria elimination and eradication. The samples are relatively easy to collect and can be used by surveillance programmes to detect evidence of malaria transmission, especially in low resource settings since the number of samples that need to be tested is reduced. Finally, as evidenced by studies of other mosquito-borne diseases, it appears that excretion of pathogens by infected mosquitoes is a general phenomenon that can be exploited for research and surveillance applications. 


\section{Additional files}

Additional file 1: Figure S1. Kaplan-Meier survival curves for cohorts of mosquitoes exposed to five different gametocyte cultures. The survival distribution was not different between cohorts (Log-Rank statistic $\chi_{(3)}^{2}=4.415, P=0.220$ ).

Additional file 2: Figure S2. RT-rtPCR standard curve. The standard curve was prepared using a suspension of $P$. falciparum sporozoites isolated from mosquito salivary glands. $X$-axis corresponds to the concentration of triplicate serially diluted template; $Y$-axis corresponds to RT-rtPCR $C_{t}$ values.

\section{Abbreviations}

ACT: artemisinin-based combination therapy; ELISA: enzyme-linked immunosorbent assay; PCR: polymerase chain reaction; PE: post-exposure; FP: filter paper; RT-rtPCR: real-time reverse transcription polymerase chain reaction; RDT: rapid diagnostic test; malERA: malaria Eradication Research Agenda.

\section{Acknowledgements}

The authors wish to thank the members of the Infection and Immunity Division at the Walter and Eliza Hall Institute of Medical Research, especially Melissa Hobbs, Charlie Jennison, Matthew O'Neill, and Sash Lopaticki for their assistance in various aspects of parasite maintenance, mosquito rearing and infection. The authors also thank Amanda de Jong and Neelima Nair for their technical assistance, and Frederick Moore for advice. The authors thank Michael Townsend for the preparation of the modified containers, Claire Wang for her advice in optimizing the nucleic acid extraction and Karen Hawkins for providing materials for parasite staining and visualization.

\section{Authors' contributions}

ALR and SME performed the experiments. ALR, GRH and JLM analyzed the samples. AFvdH, JAB, ASPY and SME provided the study materials. ALR, AFvdH, SAR and SME conceptualized the study. IMM developed the molecular assay. ALR performed the data analyses. ALR wrote the initial draft of the manuscript. $S A R, J A B$ and $A F v d H$ acquired the funding for the research. All authors read and approved the final manuscript.

\section{Funding}

This work was supported by a Queensland Government funded Australian Institute of Tropical Health and Medicine Capacity Building Grant. Insectary support was provided by the Walter and Eliza Hall Institute, NHMRC Project Grant 1049811 and a Victorian State Government Operational Infrastructure Support and Australian Government NHMRC IRIISS. ALR was supported by a doctoral scholarship granted by the Australian Institute of Tropical Health and Medicine, James Cook University QLD, Australia. JAB and SAR were supported, in part, by National Health and Medical Research Council research fellowships 1123727 and 1044698, respectively. The funders had no role in study design, data collection and analysis, decision to publish, or preparation of the manuscript.

\section{Availability of data and materials}

All data generated or analyzed during this study are included in this published article and its additional files.

\section{Ethics approval and consent to participate}

Protocols involving mice were conducted in strict accordance with the recommendations in the National Statement on Ethical Conduct in Animal Research of the National Health and Medical Research Council and were reviewed and approved by the Walter and Eliza Hall Institute of Medical Research Animal Ethics Committee (AEC2014.030).

\section{Consent for publication}

Not applicable.

\section{Competing interests}

The authors declare that they have no competing interests.

\section{Author details}

${ }^{1}$ College of Public Health, Medical and Veterinary Sciences, James Cook University, PO Box 6811, Cairns, QLD 4870, Australia. ${ }^{2}$ Australian Institute of Tropical Health and Medicine, James Cook University, PO Box 6811, Cairns, QLD 4870, Australia. ${ }^{3}$ Public Health Virology, Forensic and Scientific Services, Department of Health, Queensland Government, Coopers Plains, QLD 4108, Australia. ${ }^{4}$ Infection and Immunity Division, The Walter and Eliza Hall Institute of Medical Research, Parkville, VIC 3052, Australia. ${ }^{5}$ Department of Medical Biology, The University of Melbourne, Parkville, VIC 3052, Australia. ${ }^{6}$ Present Address: Department of Medical Microbiology Parasitology, Radboud University Medical Center, Geert Grooteplein 28, Microbiology 268, 6500 HB Nijmegen, The Netherlands.

Received: 22 January 2019 Accepted: 8 July 2019

Published online: 18 July 2019

\section{References}

1. WHO. World malaria report 2018. World Health Organization. 2018; https ://www.who.int/malaria/publications/world-malaria-report-2018/en/. Accessed 10 May 2019.

2. Olliaro P. Mortality associated with severe Plasmodium falciparum malaria increases with age. Clin Infect Dis. 2008;47:158-60.

3. Hay SI, Sinka ME, Okara RM, Kabaria CW, Mbithi PM, Tago CC, et al. Developing global maps of the dominant anopheles vectors of human malaria. PLoS Med. 2010;7:e1000209.

4. Sinka ME, Bangs MJ, Manguin S, Rubio-Palis Y, Chareonviriyaphap T, Coetzee $M$, et al. A global map of dominant malaria vectors. Parasit Vectors. 2012;5:69.

5. WHO. Eliminating malaria. Geneva: World Health Organization; 2016. https://www.who.int/malaria/publications/atoz/eliminating-malaria/en/. Accessed 10 May 2019

6. Rabinovich RN, Drakeley C, Djimde AA, Hall BF, Hay SI, Hemingway J, et al. malERA: an updated research agenda for malaria elimination and eradication. PLoS Med. 2017;14:e1002456.

7. WHO. Global technical strategy for malaria 2016-2030. Geneva: World Health Organization; 2015. https://www.who.int/malaria/publications/ atoz/9789241564991/en/. Accessed 10 May 2019.

8. WHO. Malaria surveillance, monitoring and evaluation: a reference manual. Geneva: World Health Organization; 2018. https://www.who.int/ malaria/publications/atoz/9789241565578/en/. Accessed 10 May 2019.

9. Habluetzel A, Merzagora L, Jenni L, Betschart B, Rotigliano G, Esposito F. Detecting malaria sporozoites in live, field-collected mosquitoes. Trans $\mathrm{R}$ Soc Trop Med Hyg. 1992;86:138-40.

10. Beier JC, Onyango FK, Ramadhan M, Koros JK, Asiago CM, Wirtz RA, et al. Quantitation of malaria sporozoites in the salivary glands of wild Afrotropical Anopheles. Med Vet Entomol. 1991;5:63-70.

11. Wirtz RA, Burkot TR, Graves PM, Andre RG. Field evaluation of enzymelinked immunosorbent assays for Plasmodium falciparum and Plasmodium vivax sporozoites in mosquitoes (Diptera: Culicidae) from Papua New Guinea. J Med Entomol. 1987;24:433-7.

12. Burkot TR, Zavala F, Gwadz RW, Collins FH, Nussenzweig RS, Roberts DR. Identification of malaria-infected mosquitoes by a two-site enzymelinked immunosorbent assay. Am J Trop Med Hyg. 1984;33:227-31.

13. Ryan JR, Dav K, Emmerich E, Garcia L, Yi L, Coleman RE, et al. Dipsticks for rapid detection of Plasmodium in vectoring Anopheles mosquitoes. Med Vet Entomol. 2001;15:225-30.

14. Ryan JR, Dave K, Collins KM, Hochberg L, Sattabongkot J, Coleman RE, et al. Extensive multiple test centre evaluation of the VecTest ${ }^{\mathrm{TM}}$ malaria antigen panel assay. Med Vet Entomol. 2002;16:321-7.

15. Appawu MA, Bosompem KM, Dadzie S, McKakpo US, Anim-Baidoo I, Dykstra E, et al. Detection of malaria sporozoites by standard ELISA and VecTest ${ }^{\mathrm{TM}}$ dipstick assay in field-collected anopheline mosquitoes from a malaria endemic site in Ghana. Trop Med Int Health. 2003;8:1012-7.

16. Echeverry DF, Deason NA, Makuru V, Davidson J, Xiao H, Niedbalski J, et al. Fast and robust single PCR for Plasmodium sporozoite detection in mosquitoes using the cytochrome oxidase I gene. Malar J. 2017;16:230.

17. Chaumeau V, Andolina C, Fustec B, Tuikue Ndam N, Brengues C, Herder $\mathrm{S}$, et al. Comparison of the performances of five primer sets for the 
detection and quantification of Plasmodium in anopheline vectors by real-time PCR. PLoS ONE. 2016;11:e0159160.

18. Calzetta M, Perugini E, Seixas G, Sousa CA, Guelbeogo WM, Sagnon N, et al. A novel nested polymerase chain reaction assay targeting Plasmodium mitochondrial DNA in field-collected Anopheles mosquitoes. Med Vet Entomol. 2018;32:372-7.

19. Beier JC, Perkins PV, Koros JK, Onyango FK, Gargan TP, Wirtz RA, et al. Malaria sporozoite detection by dissection and ELISA to assess infectivity of afrotropical Anopheles (Diptera: Culicidae). J Med Entomol. 1990;27:377-84.

20. Durnez L, Van Bortel W, Denis L, Roelants P, Veracx A, Trung HD, et al. False positive circumsporozoite protein ELISA: a challenge for the estimation of the entomological inoculation rate of malaria and for vector incrimination. Malar J. 2011;10:195.

21. Rosenberg R, Wirtz RA, Schneider I, Burge R. An estimation of the number of malaria sporozoites ejected by a feeding mosquito. Trans R Soc Trop Med Hyg. 1990;84:209-12.

22. Hall-Mendelin S, Ritchie SA, Johansen CA, Zborowski P, Cortis G, Dandridge $S$, et al. Exploiting mosquito sugar feeding to detect mosquitoborne pathogens. Proc Natl Acad Sci USA. 2010;107:11255-9.

23. van den Hurk AF, Hall-Mendelin S, Townsend M, Kurucz N, Edwards J, Ehlers $\mathrm{G}$, et al. Applications of a sugar-based surveillance system to track arboviruses in wild mosquito populations. Vector Borne Zoonotic Dis. 2014;14:66-73.

24. Brugman VA, Kristan M, Gibbins MP, Angrisano F, Sala KA, Dessens JT, et al. Detection of malaria sporozoites expelled during mosquito sugar feeding. Sci Rep. 2018:8:7545.

25. Fontaine A, Jiolle D, Moltini-Conclois I, Lequime S, Lambrechts L. Excretion of dengue virus RNA by Aedes aegypti allows non-destructive monitoring of viral dissemination in individual mosquitoes. Sci Rep. 2016;6:24885.

26. Ramírez AL, Hall-Mendelin S, Doggett SL, Hewitson GR, McMahon JL, Ritchie SA, et al. Mosquito excreta: a sample type with many potential applications for the investigation of Ross River virus and West Nile virus ecology. PLoS Negl Trop Dis. 2018;12:e0006771.

27. Meyer DB, Ramírez AL, van den Hurk AF, Kurucz N, Ritchie SA. Development and field evaluation of a system to collect mosquito excreta for the detection of arboviruses. J Med Entomol. 2019;56:1116-321.

28. Pilotte N, Zaky WI, Abrams BP, Chadee DD, Williams SA. A novel xenomonitoring technique using mosquito excreta/feces for the detection of filarial parasites and malaria. PLoS Negl Trop Dis. 2016;10:e0004641.

29. Cook DAN, Pilotte N, Minetti C, Williams SA, Reimer LJ. A superhydrophobic cone to facilitate the xenomonitoring of filarial parasites, malaria, and trypanosomes using mosquito excreta/feces. Gates Open Res. 2017;1:7.

30. Yang ASP, O'Neill MT, Jennison C, Lopaticki S, Allison CC, Armistead JS, et al. Cell traversal activity is important for Plasmodium falciparum liver infection in humanized mice. Cell Rep. 2017:18:3105-16.

31. Saliba KS, Jacobs-Lorena M. Production of Plasmodium falciparum gametocytes in vitro. Methods Mol Biol. 2013;923:17-25.

32. MCCarthy JS, Griffin PM, Sekuloski S, Bright AT, Rockett R, Looke D, et al. Experimentally induced blood-stage Plasmodium vivax infection in healthy volunteers. J Infect Dis. 2013;208:1688-94.

33. Gooding RH. Digestive processes of haematophagous insects. I. A literature review. Quaest Entomol. 1972;8:5-60.

34. Baton LA, Ranford-Cartwright LC. Spreading the seeds of million-murdering death: metamorphoses of malaria in the mosquito. Trends Parasitol. 2005;21:573-80.

35. Eichner M, Diebner HH, Molineaux L, Collins WE, Jeffery GM, Dietz K. Genesis, sequestration and survival of Plasmodium falciparum gametocytes: parameter estimates from fitting a model to malariatherapy data. Trans $\mathrm{R}$ Soc Trop Med Hyg. 2001;95:497-501.

36. Beier JC. Malaria parasite development in mosquitoes. Annu Rev Entomol. 1998;43:519-43.
37. Meis JFGM, Wismans PGP, Jap PHK, Lensen AHW, Ponnudurai T. A scanning electron microscopic study of the sporogonic development of Plasmodium falciparum in Anopheles stephensi. Acta Trop. 1992;50:227-36.

38. Rosenberg R, Rungsiwongse J. The number of sporozoites produced by individual malaria oocysts. Am J Trop Med Hyg. 1991;45:574-7.

39. Hillyer JF, Barreau C, Vernick KD. Efficiency of salivary gland invasion by malaria sporozoites is controlled by rapid sporozoite destruction in the mosquito haemocoel. Int J Parasitol. 2007;37:673-81.

40. Beier JC, Davis JR, Vaughan JA, Noden BH, Beier MS. Quantitation of Plasmodium falciparum sporozoites transmitted in vitro by experimentally infected Anopheles gambiae and Anopheles stephensi. Am J Trop Med Hyg. 1991;44:564-70.

41. Stone WJR, Eldering M, van Gemert G-J, Lanke KHW, Grignard L, van de Vegte-Bolmer MG, et al. The relevance and applicability of oocyst prevalence as a read-out for mosquito feeding assays. Sci Rep. 2013;3:3418.

42. Murdock CC, Blanford S, Luckhart S, Thomas MB. Ambient temperature and dietary supplementation interact to shape mosquito vector competence for malaria. J Insect Physiol. 2014;67:37-44.

43. Ohm JR, Baldini F, Barreaux P, Lefevre T, Lynch PA, Suh E, et al. Rethinking the extrinsic incubation period of malaria parasites. Parasit Vectors. 2018;11:178.

44. Spence PJ, Jarra W, Levy P, Nahrendorf W, Langhorne J. Mosquito transmission of the rodent malaria parasite Plasmodium chabaudi. Malar J. 2012:11:407.

45. Amino R, Thiberge S, Martin B, Celli S, Shorte S, Frischknecht F, et al. Quantitative imaging of Plasmodium transmission from mosquito to mammal. Nat Med. 2006;12:220-4.

46. Walliker D, Quakyi IA, Wellems TE, McCutchan TF, Szarfman A, London WT, et al. Genetic analysis of the human malaria parasite Plasmodium falciparum. Science. 1987;236:1661-6.

47. Collins WE. Animal models. I. Infection of monkeys with Plasmodium spp. In: Moll K, Kaneko A, Scherf A, Wahlgren M, editors. Methods in malaria research. 6th ed. Glasgow: EVIMalaR; 2013. p. 169-71.

48. Flies EJ, Toi C, Weinstein P, Doggett SL, Williams CR. Converting mosquito surveillance to arbovirus surveillance with honey-baited nucleic acid preservation cards. Vector Borne Zoonotic Dis. 2015;15:397-403.

49. Steiner CD, Riemersma KK, Stuart JB, Singapuri A, Lothrop HD, Coffey LL. Scented sugar baits enhance detection of St. Louis encephalitis and West Nile viruses in mosquitoes in suburban California. J Med Entomol. 2018:55:1307-18.

50. Jeanne I, Chambers LE, Kazazic A, Russell TL, Bobogare A, Bugoro H, et al. Mapping a Plasmodium transmission spatial suitability index in Solomon Islands: a malaria monitoring and control tool. Malar J. 2018;17:381.

51. WHO, FIND, CDC. Malaria rapid diagnostic test performance: summary results of WHO product testing of malaria RDTs: rounds 1-6 (2008-2015). Geneva: World Health Organization; 2015. https://www.who.int/malaria/ publications/atoz/9789241510035/en/. Accessed 10 May 2019.

52. Vontas J, Mitsakakis K, Zengerle R, Yewhalaw D, Sikaala CH, Etang J, et al. Automated innovative diagnostic, data management and communication tool, for improving malaria vector control in endemic settings. Stud Health Technol Inform. 2016;224:54-60.

53. malERA Refresh Consultative Panel on Characterising the Reservoir and Measuring Transmission. malERA: an updated research agenda for characterising the reservoir and measuring transmission in malaria elimination and eradication. PLoS Med. 2017;14:e1002452.

\section{Publisher's Note}

Springer Nature remains neutral with regard to jurisdictional claims in published maps and institutional affiliations. 